\title{
Carnets
}

Revue électronique d'études françaises de l'APEF

Deuxième série - 7| 2016

Plurilinguisme et migrations dans la littérature de langue française

\section{Écrire (entre) ses langues en atelier d'écriture}

Une approche plurilingue des textes de Driss Chraib, Wajdi Mouawad et Nancy Huston

\section{Noëlle Mathis}

\section{(2) OpenEdition}

Journals

Édition électronique

URL : http://journals.openedition.org/carnets/1080

DOI : $10.4000 /$ carnets. 1080

ISSN : 1646-7698

Éditeur

APEF

Référence électronique

Noëlle Mathis, «Écrire (entre) ses langues en atelier d'écriture », Carnets [En ligne], Deuxième série -

7 | 2016, mis en ligne le 31 mai 2016, consulté le 19 avril 2019. URL : http://journals.openedition.org/ carnets/1080; DOI : 10.4000/carnets.1080

Ce document a été généré automatiquement le 19 avril 2019.

\section{(c) (i) (8)}

Carnets est mis à disposition selon les termes de la licence Creative Commons - Atribution - Pas d'utilisation commerciale 4.0 International. 


\section{Écrire (entre) ses langues en atelier d'écriture}

Une approche plurilingue des textes de Driss Chraib, Wajdi Mouawad et Nancy Huston

\section{Noëlle Mathis}

1 Dans le cadre d'ateliers d'écriture plurilingue avec des apprenants adultes de français langue étrangère (FLE), j'utilise des extraits de textes d'auteurs qui ont la particularité d'adopter le français comme langue principale, mais non exclusive, d'écriture, tels Driss Chraïbi (1998), Wajdi Mouawad (2008) et Nancy Huston (1999). Plusieurs raisons motivent mon choix : outre le choix de(s) langue(s) d'écriture, les auteurs partagent avec leurs lecteurs certaines de leurs représentations au sujet des langues et des cultures par le biais de leur expérience personnelle et interrogent leurs langues en mettant en exergue leur « surconscience linguistique » (Gauvin, 2000) et en jouant de leur répertoire plurilingue (Coste, Moore \& Zarate, 1997, 2009; Moore, 2006). Au croisement des champs disciplinaires de la didactique des langues et du plurilinguisme et de la littérature plurilingue, je tente de comprendre comment la mise en évidence du répertoire plurilingue d'un auteur, par le biais d'analyses de textes en classe, peut servir de tremplin à des apprenants-scripteurs plurilingues, en les autorisant à utiliser leur palette de langues lors de productions écrites et d'exprimer leurs identités linguistiques.

2 Ainsi, dans un contexte de création textuelle en plusieurs langues, au sein d'une salle de classe à l'université, je me pose les questions suivantes. Quand une fenêtre créative plurilingue est offerte à des apprenants de langues, qu'en font-ils dans leurs textes? Comment le répertoire plurilingue d'auteurs peut-il influencer l'écriture des apprenants? Comment se manifestent les identités plurilingues des apprenants-scripteurs dans leurs textes?

Dans cet article, je présenterai successivement le contexte didactique des ateliers d'écriture plurilingue et ce que j'entends par personne plurilingue, notamment en lien avec le répertoire et les compétences plurilingues et plurilittératiées ${ }^{1}$. Puis, je définirai les écrits plurilingues selon une approche sociolinguistique en mettant en exergue les stratégies possibles, dont le tissage codique. Enfin, je proposerai une analyse des textes de 
Chraïbi, Mouawad et Huston, suivie d'une présentation et de commentaires portant sur des textes d'apprenants.

\section{Un atelier d'écriture plurilingue}

$4 \quad$ Les ateliers d'écriture plurilingue que j'anime sont caractérisés par plusieurs facteurs. Premièrement, ils s'inscrivent dans une «approche didactique plurielle » qui met «en œuvre des activités impliquant à la fois plusieurs variétés linguistiques et culturelles " (Candelier, 2008: 68). Plus précisément, les ateliers sont conçus en prenant en compte le fait que les apprenants sont plurilingues. Ainsi, les approches didactiques plurielles s'adressent-elles à l'ensemble des apprenants, et notamment ceux qui possèdent dans leur répertoire langagier plusieurs langues autres que celles de l'école; elles permettent la mise en relation des langues dans un processus d'ouverture et d'intégration; elles font appel à la diversité des langues qui sont « déjà-là » sans l'ambition de les faire apprendre et sans accorder d'importance à l'une plus qu'à l'autre ; et enfin, elles concernent à la fois des aptitudes langagières et métalangagières.

5 Deuxièmement, les ateliers d'écriture plurilingues menés avec des apprenants de FLE ont, avant tout, pour objectif de faire écrire (et dire) à des apprenants des fragments biographiques de leurs parcours de mobilité et de leur faire découvrir leur(s) voix. Les écrits sont des identity texts, nommés ainsi par Cummins \& Early (2011), c'est-à-dire des textes écrits, oraux et / ou performés, créés par un apprenant au sein d'un espace pédagogique et orchestré par l'enseignant. Les apprenants y investissent leurs identités en combinant différents modes d'écrits, de visuels sous des formes multimodales. Précisons que, dans l'atelier d'écriture, au vu des pratiques langagières, sont principalement mises en lumière des "identités plurilingues ${ }^{2}$ " (Moore \& Brohy, 2013). Selon Cummins \& Early, an identity text « holds a mirror up to students in which their identities are reflected in a positive light » (2011: 3). De plus, lorsque les apprenants partagent leur identity texts avec une audience sous formes diverses (lecture à haute voix en classe, recueil de textes, lecture publique), ils sont susceptibles de recevoir des feedback positifs et une affirmation de soi lors de, et après, l'interaction avec ce public.

6 Troisièmement, l'atelier d'écriture plurilingue prend son inspiration dans des récits ou processus narratifs d'origines différentes. J'en considère trois: les premiers, ceux des auteurs publiés qui servent de base de discussion en classe. Ceux de Chraïbi (1998), Mouawad (2008) et Huston (1999) font l'objet de l'analyse de cet article. Les deuxièmes, ceux qui concernent mon propre parcours en tant que migrante plurilingue ayant vécu l'exil et la condition d'étrangère (voir Mathis, 2014). Les troisièmes, ceux des apprenants, de nature très variés, à la fois fragments de leurs expériences, textes qu'ils écrivent et réécrivent au cours de la session, récits oraux de leurs parcours. L'ensemble de ces récits, ayant une forme et une fonction d'étayage, offrent un pont entre l'expérience narrativisée des uns et celle, à faire émerger, formuler et interpréter, des autres. En d'autres termes, les récits offrent un tremplin entre des connaissances « déjà-là » et celles nouvelles qui peuvent ainsi naître, balbutier et progressivement croître. 


\section{Des personnes bi-/plurilingues}

7 Les apprenants de l'étude sont considérées comme des personnes bi-/plurilingues, comme l'entend Grosjean c'est-à-dire des « personnes qui se servent de deux ou plusieurs langues (ou dialectes) dans la vie de tous les jours » (Grosjean, 1995 : 14), et - aspect essentiel - qui maîtrisent leurs langues et leurs variétés de langues à des degrés différents. Certains pourront avoir une compétence développée à lire dans une langue alors qu'écrire dans cette même langue posera plus de difficultés ; d'autres auront recours à l'écriture comme moyen d'expression alors que leur production orale reflètera leur hésitation. De plus, outre les compétences à des niveaux variés, l'individu plurilingue utilise également ses différentes langues sur un continuum et peut donc passer d'un mode monolingue à un mode bilingue. Il peut, par ailleurs, passer d'une posture d'apprenant à celle d'expert dans des situations différentes quand le besoin se présente.

8 On peut donc dire que les personnes bi-/plurilingues font usage d'un répertoire plurilingue (Coste, Moore \& Zarate, 1997, 2009; Moore, 2006) composé de l'ensemble des langues et de leurs variétés linguistiques utilisées selon les situations de communication spécifiques et en fonction des interlocuteurs. Ce répertoire plurilingue inclurait les différentes compétences à des degrés variés dans différentes langues sur un continuum en fonction de la situation de communication, des interlocuteurs en présence (ou en absence), des intentions de communication, mais aussi selon "les configurations identitaires que les discours permettent de prendre en charge localement »(Moore, 2006 : 100).

9 Les personnes bi-/plurilingues sont également dotées d'une compétence plurilingue et pluriculturelle (Coste, Moore et Zarate, 1997, 2009), considérée comme un ensemble global de ressources langagières et de mises en relation «au sein d'une compétence conçue comme globale (englobante), dynamique (susceptible de reconfigurations et d'évolutions suivant un parcours de vie), singulière (différente pour chaque individu), et porteuse de valeurs symboliques et identitaires " (Moore, $2006: 213$ ). Son caractère global et non segmenté est essentiel : la compétence plurilingue n'est pas considérée comme la somme des compétences à communiquer dans différentes langues mais comme une compétence complexe et unique qui englobe l'ensemble du répertoire langagier à disposition (Candelier, 2008). Par ailleurs, se joint à cette notion « des compétences de type plurilittératiées » (Dagenais \& Moore, 2008: 15), qui prennent en compte les façons complexes et multiples qu'une personne possède pour donner du sens à travers ses pratiques d'écriture en plusieurs langues.

\section{Les écrits plurilingues}

10 Les écrits plurilingues ont été récemment définis dans les sciences humaines par Van den Avenne (2013) qui envisage quatre types dans lesquels s'inscrivent des phénomènes différents de contacts de langues: les textes translittérés, les textes mixtes, les textes bilingues, les textes reflétant implicitement une situation bilingue. Avant de les définir, je tiens cependant à justifier la prise en compte d'une notion émanant du champ de la sociolinguistique plutôt que de la littérature ou des études littéraires. En effet, on pourrait se référer aux travaux de Dion, Lüsebrink \& Riesz (2002) pour distinguer les différentes « stratégies de détour » à disposition des auteurs plurilingues, soit « le passage 
d'une langue à l'autre ", « l'écriture de deux ou plusieurs langues à la fois », « l'alternance des langues». Toutefois, se présentent deux défis majeurs. Le premier consiste en la présence très forte, dans le domaine de la littérature et de la critique littéraire, d'une (dé)limitation importante entre ce que constituent langues maternelles et langues étrangères et, par conséquent, l'usage de notions telle que l'interférence qui n'est plus utilisée dans la conception actuelle du plurilinguisme. Le deuxième est, de ce fait, qu'il ne prend pas en compte ni le répertoire plurilingue de l'auteur et la possibilité propre à toute personne bi-/plurilingue de naviguer entre langues et discours, comme explicité dans la section précédente, ni le fait que l'auteur pourrait avoir plusieurs langues maternelles.

11 Revenons à Van den Avenne. Ses deux premiers types d'écrits plurilingues conviennent partiellement pour décrire les écrits des auteurs étudiés en classe et les textes écrits par les apprenants. En effet, les textes translittérés « sont des textes écrits dans une graphie 'importée' d'une langue différente de celle dans laquelle est écrite le texte » (Van den Avenne, 2013 : 248). De tels textes mettent en valeur le fait qu'une langue peut être écrite dans une graphie autre que celle dans laquelle elle est écrite dans son contexte d'origine (l'arabe écrit en alphabet latin par exemple). L'utilisation d'une autre graphie soulignera un rapport de pouvoir par rapport à la norme de la langue dans laquelle le texte est écrit. Les textes mixtes, quant à eux, "sont caractérisés par des changements de langue en cours de texte, c'est-à-dire des phénomènes d'alternance codique et de mélange de codes" (ibid.: 249). Ils seraient caractérisés par l'utilisation de plusieurs codes linguistiques et registres de langues qui se manifeste par l'emprunt, l'alternance, entre autres, tout en sachant qu'en général ils sont marqués par une langue de base et que l'alternance, par exemple, peut ne concerner que de brèves insertions.

Une perspective supplémentaire à apporter aux écrits plurilingues serait celle du tissage codique (code-meshing) défini par Canagarajah $(2006,2011)$ comme une stratégie de communication plurilingue qui, ni ne sépare les codes linguistiques et leurs utilisations en fonction des contextes appropriés, ni ne suggère une hiérarchisation entre plusieurs codes, comme le ferait l'alternance codique. Canagarajah précise qu'il s'agit, pour le scripteur plurilingue, de "shuttle between languages, treating the diverse languages that form their repertoire as an integrated system " (Canagarajah, 2011 : 401). En d'autres termes, le tissage codique fait référence à la fusion de codes et à leur utilisation dans le même texte, et ce, ajoute Canagarajah (2006), afin de servir les intérêts des apprenants et de valoriser leurs identités plurielles. Sont donc remises en question les frontières des langues. De plus, à la dimension des codes linguistiques, Canagarajah ajoute la dimension multimodale, pour les apprenants-scripteurs, qui leur permet d'utiliser, parmi les mots écrits, d'autres modes de communication tels que les images, les photos, les symboles, les espaces, les icones. Si Canagarajah propose le code-meshing pour l'écriture au sein d'une salle de classe, il s'applique à tout écrit par tout scripteur faisant partie, ou ayant navigué, entre des communautés multilingues. Ce phénomène n'étant pas nouveau, Canagarajah suggère que les apprenants provenant de communautés multilingues puissent s'inspirer des traditions de littératies multilingues et multimodales qui leur sont proposés de par leur héritage culturel et traditionnel.

13 Par ailleurs, l'écrit plurilingue est également un écrit dialogique. Dans l'atelier d'écriture, je conçois, en effet, les textes des auteurs retenus à la fois comme pré-textes au sens littéral du terme - ceux qui précèdent le texte - et comme prétextes à l'écriture des apprenants du groupe. En effet, les textes d'auteurs font l'objet d'interactions orales en 
classe durant lesquelles, non seulement sont discutés le contenu du texte, le style d'écriture, les choix linguistiques, mais aussi permis, entre ces analyses textuelles, des récits d'expérience personnelle de mobilité et d'altérité des apprenants, entremêlés de leurs langues autres que la langue cible. Je considère donc les textes d'auteurs et les interactions comme des pré-textes. Et ce qui précède le texte demande réponse : le prétexte est un prétexte au dialogue. Il demande réponse, et celle-ci se manifeste et se concrétise dans le texte écrit par les apprenants qui (re)prend certaines des discussions en classe, et aussi certains des procédés linguistiques utilisés par les auteurs selon le principe d'intertextualité.

14 En utilisant des textes qui offrent un miroir de la réalité des apprenants plurilingues, on retrouve ce que Cicurel appelle « le principe de réciprocité » dans l'acte de lecture, selon lequel le texte est "envisagé comme une partition dont les lecteurs seraient les interprètes » (Cicurel, $2000: 262$ ). Ce qui m'intéresse, c'est le décodage auquel procède le lecteur qui lui permet de construire une « figure énonciative » à travers laquelle il peut se reconnaître (ou non). C'est pourquoi, dans ce qui suit, je souhaite me pencher sur les stratégies textuelles que Chraïbi, Mouawad et Huston ont mises en œuvre pour représenter la langue. Je chercherai ainsi à examiner comment le texte parle la langue et comment la langue parle de l'auteur. Si j'analyse ces textes et les procédés d'écriture mis en place, c'est parce que je tente de faire un lien entre ce que font les auteurs que nous avons étudiés en classe et les stratégies textuelles que les apprenants plurilingues ont déployées dans leurs propres textes, les uns ayant influencé les autres de manière parfois tout à fait inconsciente.

\section{Les textes des auteurs}

\section{L'écrit plurilingue de Driss Chraïbi}

15 Né au Maroc en 1926, Chraïbi se consacre entièrement à l'écriture à partir de 1952. Les thèmes abordés dans ses romans sont le choc des cultures, l'oppression des femmes et des enfants dans la société patriarcale et la confrontation entre les mondes ruraux et urbains. L'extrait de $V u$, lu, entendu (1998) étudié en classe met en exergue le récit d'un narrateurauteur relatant son apprentissage du français à l'âge de six ans. Le texte retrace ses représentations des langues de son répertoire plurilingue : l'arabe, le français et l'anglais et fait ressortir une série d'opposition et de tension entre les trois langues. Cependant, il s'agit de remarquer qu'il s'agit de trois langues apprises à l'école, qui seraient considérées comme des langues normées : l'arabe (certainement classique), le français et l'anglais. Le narrateur-auteur ne fait pas mention d'autre(s) langue(s) qu'il parlait dans sa famille en tant qu'enfant et qui ne serai(en)t aucune de ces trois.

D’abord, la langue française est décrite comme étant «sous-développée » : il lui manque des lettres et des sons que possède l'arabe. L'altérité que représente la langue française est mise en valeur par l'italique: il s'agit d'une "autre langue». La perte de sens et la confusion ressenties par l'enfant, face à cette autre langue ayant un alphabet latin et s'écrivant selon une pratique de littératie nouvelle, sont signifiées grâce à des termes géographiques : «le planisphère (...) représentait le globe terrestre à l'envers », « l'Europe en haut et l'Afrique en bas", "L'Orient à droite et l'Océan Atlantique à gauche " accompagnés de termes exprimant l'opposition: "ce devrait être le contraire", "à l'envers ", "l'inverse du nôtre » et également des réflexions sur les transformations 
physiques: "je devins gaucher", "ma tête a commencé à tourner». Mais surtout, l'auteur choisit une pratique d'écriture translittérée en écrivant de droite à gauche son nom comme il le ferait en arabe : "ssird tse mon noM", évoque la notion de "miroir » nécessaire pour « rétablir la phrase dans le bon sens : Mon nom est Driss ». Miroir qui luimême est mis en abîme avec le possessif « mon » qui se reflète en " nom ». Dans les deux cas, la phrase simple d'introduction de soi est écrite en italique : utilisant la langue française, son monde s'est profondément transformé et l'a profondément transformé, dans son identité linguistique, altérant son regard sur lui-même, son peuple et les cultures.

Puis, la langue arabe dans le texte s'inscrit dans un désir de transmettre : "madrassa = école, koursille = chaise, calame = plume, midad = encre $»$ comme le font les enseignants $/$ médiateurs de langue(s), mais surtout pour critiquer le pouvoir attribué à la langue du colonisateur. En effet, son choix de l'alphabet latin, de l'italique et du signe mathématique égal sont sujets à réflexion: l'alphabet latin est utilisé pour des mots d'arabe alors qu'il aurait pu choisir d'écrire dans l'alphabet et la graphie arabe. De plus, ces mêmes mots sont écrits en italique, pour désigner à la fois leur caractère étranger pour lui du mot arabe étant écrit non seulement en alphabet latin, mais aussi en français. Enfin, le signe égal tenterait d'établir une certaine équivalence en mettant l'arabe et le français sur le même pied d'égalité. Ce serait la représentation monolingue qu'une langue est équivalente à l'autre en mettant en correspondance leurs dictionnaires. Cependant, si Chraïbi justement joue par le biais de ses pratiques plurilittératiées, c'est pour signaler le poids de la colonisation française qui est passé en outre par la langue avec, comme il le souligne dans ce même texte, "le vocabulaire de nos 'protecteurs' d'outreMéditerranée », en utilisant les guillemets autour de protecteurs.

Enfin l'auteur, avec beaucoup d'humour, évoque l'influence du français sur l'anglais : « je récitai (...) avec la prononciation française : Thé ski is blu (The sky is blue)... i âme Driss (I am Driss) ». Ce faisant, il manifeste, dans l'apprentissage de l'anglais, l'utilisation des accents (bien français de France), soulignant ainsi (et se moquant peut-être) des Français et de leurs compétences à parler l'anglais. Toutefois, en tant qu'écrivain extrêmement habile de son répertoire plurilingue et plurilittératié, il parle à son lecteur de «thé » et d'« âme » qui évoqueraient l'arabe, tout en jouant de ce que Canagarajah (2006) appelle le code-meshing: Chraïbi ne traduit pas l'anglais qu'il utilise pour marquer l'altérité prosodique, mais l'italique pour indiquer la prononciation à la française, puis entre parenthèses et sans signe égal, la même phrase en anglais corrigé est écrit sans italique, tout en transmettant en français deux mots symbolisant l'arabe. Par ailleurs, il évoque dans son texte le fait que ses camarades l'ont longtemps appelé «l'Angliche». Je vois deux raisons pour lesquelles il aurait fait ce choix. D'une part, en utilisant ce mot d'argot, composé de la première syllabe du mot "anglais» et de la terminaison -ish du mot " english » prononcé à la française, assigné en général par un Français à un locuteur britannique, il change complètement de registre de langue. De ce fait, par l'adoption d'une prosodie française (de France), il s'identifie plus à un locuteur-scripteur français qu'à un scripteur de langue arabe, jouant ainsi de ses identités grâce à son répertoire plurilingue et plurilittératié. D'autre part, dans le mot « Angliche », on entend également la reconnaissance d'un statut de locuteur plurilingue expert, dans une langue non partagée par les autres locuteurs, qui le mettent à la fois à distance et à proximité, de par sa différence, car en l'imitant ils s'en rapprochent. 


\section{La perte de la langue maternelle de Wajdi Mouawad}

19 Wajdi Mouawad est né au Liban en 1968. Avec sa famille, il quitte son pays natal à l'âge de onze ans et immigre en France puis à Montréal en 1983. Homme de théâtre, il écrit principalement des textes qui sont mis en scène. Le texte étudié en atelier d'écriture «la langue maternelle » est un extrait du livre Seuls (2008) constitué de réflexions autour de l'écriture, notamment celle de la genèse du texte Seuls dans lequel un fils essaie de communiquer avec son père, tous deux exilés.

20 Le thème du texte est la perte de la langue maternelle, perte qui se décline de plusieurs façons. D'abord, l'auteur-narrateur évoque son lien douloureux à la langue maternelle : "Je tente de dire le mot fenêtre en arabe. Le disant, je le dis de manière brisée comme tous les mots arabes que je tente de dire ». Le mot fenêtre est écrit en italique pour signifier l'impossible énonciation du mot en arabe. L'italique est précédé et succédé des verbes «dire» utilisé quatre fois, «tenter» deux fois et du mot «mot(s)» deux fois. L'impossibilité de dire, accentuée par cette répétition, est accompagnée du rire d'un membre de sa famille : « Ma sœur éclate de rire et se moque de moi ». L'auteur joue sur le son " ire » répété dans les verbes « dire » et « rire » que j'interprète comme une colère (ire) intérieure, celle de non-pouvoir, renforcée par les termes «abîme d'incompréhension », « impossible de traduire en arabe ", « incapable d'en comprendre les paroles ». Cependant, l'incompréhension ou, tout du moins, la « conviction que cette sensation est intimement liée à la langue maternelle » se manifeste par une quête qui se traduit dans une pragmatique du verbe: «je rentre chez moi », «je me replonge dans l'observation », «je note dans le cahier » « je me rends compte». La succession de verbes indique une profonde réflexivité qui aboutit à une autre action : « je me mets à écouter de la musique arabe ». Or, en évoquant la musique arabe, on ressent une profonde émotion, notamment par une utilisation à profusion du champ lexical de la musique : «chanson ", " mélodie", "refrain», " chants arabes", "chants interminables", «mélopées", «chantonnant », « paroles ». De plus, concomitant à l'évocation de cet univers musical, Mouawad écrit une phrase aussi longue que la promenade heureuse dont il s'agit ("promenade», « interminable», «bonne humeur») dans laquelle les nombreuses allitérations en « $\mathrm{m}$ » (" mélodie », " promenade », « emmenait », « montagnes », « interm

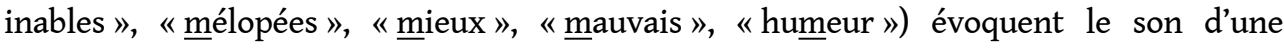
mélodie que l'on murmure à voix basse qui, dans les creux, est présente comme «le substrat d'une langue pour ainsi dire 'secrète'« (Dion et al., 2002: 12) même si la conscience ne peut en « dire les mots».

21 La perte ou la sensation de perte de la langue maternelle se traduit par une pratique d'écriture comme le suggère Robin (2003). Mouawad mentionne cette sensation dans les pages précédant l'extrait choisi, comme étant un moteur: "cette sensation à peine perceptible prendra peut-être la forme d'un spectacle ou d'un texte, mais aujourd'hui impossible de deviner l'histoire qui la porte» (Mouawad, 2008: 30). Elle lui permet d'écrire, d'être en chemin, de chercher à travers les arts, une réponse, des réponses, en ce qui concerne ses origines, des raisons pour lesquelles il ne parlerait plus la langue maternelle: «si je ne comprends plus l'arabe, c'est un peu à cause de la guerre civile (au Liban) » (ibid : 49, ma parenthèse). Enfin, il s'agira également de mentionner la dimension multimodale ajoutée à ce texte en prenant en compte l'écriture en calligraphie arabe superposée au texte écrit en lettres d'alphabet latin. En effet, l'écriture en langue 
arabe se tisse entre les lignes du texte portant sur la perte de la langue maternelle. En bref, Mouawad, sans utiliser l'alternance codique, fait surgir «le substrat» d'une autre langue et joue de ses identités plurielles, en quête d'une origine perdue mais agissant comme un fil à suivre dans l'écriture.

\section{La réflexivité de l'écriture bilingue de Nancy Huston}

Nancy Huston est née en 1953 à Calgary au Canada anglophone. Écrivaine d'expression française et anglaise, elle s'installe à Paris à l'âge de vingt ans pour y faire des études et débuter sa carrière de romancière en 1981 avec Les Variations Goldberg. Douze ans plus tard, avec le roman Cantique des plaines qu'elle écrit d'abord en anglais pour ensuite le traduire en français puisque les éditeurs de langue anglaise le refusent, elle réalise que la traduction permet d'améliorer l'original. Depuis, elle utilise cette technique de double écriture pour tous ses romans, mais se sert exclusivement du français pour ses essais et articles. Dans l'atelier d'écriture, j'ai utilisé des extraits de Nord Perdu (1999) et de Lettres parisiennes - histoires d'exil (1986) en correspondance avec Leïla Sebbar. Ces deux textes proposent des réflexions poussées sur la migration, l'exil, le passage d'une langue à l'autre. Je tiens à souligner que bien que Huston utilise les notions de langue maternelle et langue étrangère, notions largement critiquées en didactique des langues, ses textes représentent toutefois une source d'inspiration importante pour des apprenants FLE qui se questionnent sur le passage d'une langue à l'autre. Dans ce qui suit, je souhaite particulièrement me pencher sur les réflexions de Huston au sujet de l'écriture bilingue, le rapport à la langue française et certaines particularités de son style d'écriture liées à la réflexivité.

Dans la lettre VI de Lettres Parisiennes, Huston fait le récit des transformations de son journal commencé en 1970 en anglais, et treize ans plus tard entièrement en français. Elle fait le récit du changement de langue qui s'opère :

Les entrées sont tantôt en anglais, tantôt en français ; parfois la langue change d'un paragraphe à l'autre, voire à l'intérieur de la même phrase. Le processus de mutation est presque physiquement sensible à chaque page. L'un des effets de cette mutation, c'est que les italiques ont peu à peu, elles aussi changé de bord. Avant c'était les expressions françaises dans un texte anglais que je soulignais consciencieusement, et maintenant c'est l'inverse. Autrement dit, dans les pages que j'écris maintenant, ce sont les mots de ma langue maternelle qui sautent aux yeux, eux qui sont mis en valeur, eux dont le caractère exotique est systématiquement pointé (Huston \& Sebbar, 1986 : 36).

Le passage à l'autre langue lui offre une distance que sa langue maternelle ne donne pas, et lui permet d'écrire des textes que la langue maternelle n'aurait pas rendu possible :

Le plus grand vertige, en fait, s'empare de moi au moment où, ayant traduit mes propres textes - dans un sens ou dans l'autre - je me rends compte, ébahie : jamais je n'aurais écrit cela dans l'autre langue ! (Huston, 1999 : 52)

La question identitaire est permanente, notamment en ce qui concerne l'adoption d'un style d'écriture en langue française, celle-ci étant décrite par l'auteure grâce à la métaphore d'un être tout-puissant et intouchable :

Qui suis-je, en français? Je ne sais pas; tout et rien sans doute. Quand je rencontre des lycéens, ils s'étonnent souvent des ruptures de style dans mes romans, les passages abrupts du style « soutenu » au style « familier » (...). Il est plus facile pour moi étrangère que pour eux autochtones de transgresser les normes et les attentes de la langue française. C'est une très grande dame, la langue française. Une reine, 
belle et puissante. Beaucoup d'individus qui se croient écrivains ne sont que des valets à son service : ils s'affairent autour d'elle, lissent ses cheveux, ajustent ses parures, louent ses bijoux et ses atours, la flattent, et la laissent parler toute seule. Elle est intarissable, la langue française, une fois qu'elle se lance. Pas moyen d'en placer une (Huston, 1999 : 47).

Dans cet extrait, Huston questionne son identité en français (« Qui suis-je en français?») sans vraiment répondre à la question. Cependant, elle se sert de réflexions d'autrui, en l'occurrence de lycéens qui la questionnent sur son style et «ses passages abrupts du style 'soutenu' au style 'familier' «. Elle justifie cette pratique en proposant qu'il est plus facile pour une étrangère (" pour moi étrangère » est mis en opposition avec " pour eux autochtones») de détourner la langue ("transgresser les normes et les attentes de la langue française»). Être en français serait, pour elle, équivalent à avoir un style, s'autoriser à transgresser, s'écarter de la norme linguistique. Elle personnifie la langue française en lui donnant un statut de reine et en utilisant le champ lexical de la préciosité : "s'affairent autour d'elle ", « lissent ses cheveux », "ajustent ses parures », " louent ses bijoux et ses atours ", etc. Mais, le plus intéressant au niveau linguistique, à la fin de son paragraphe, l'utilisation d'une forme d'énonciation reflétant les propos mêmes de la discussion sur la langue française : " pas moyen d'en placer une ». La forme familière courte après une longue énonciation en langue soutenue dans laquelle est discutée l'autorité de la langue française en utilisant précisément cette expression crée un " passage abrupt », un écart de langue. Elle utilise donc une mise en abîme qui lui permet de mettre en action dans la langue ce qu'elle énonce. L'adoption d'un style particulier, en tant qu'écrivaine étrangère, lui donne également la possibilité de jouer avec la langue: « je commence une nouvelle phrase et aussitôt, dans ma tête, elle bifurque, trifurque: vaut-il mieux écrire est-ce que 'je cherche' ou bien 'cherché-je' ? Peut-être 'chercheraisje'« (Huston, 1999: 47). Son jeu, transformant le préfixe bi- en tri-, ne cherche pas à créer de néologismes, mais plutôt à faire surgir ce qui se joue pour les personnes bi-/ plurilingues : une personne bilingue, si elle est, en réalité, trilingue pensera peut-être à jouer avec le bi- qui pour elle est tri-. De la même façon, tout apprenant de langue française, ayant été sensibilisé à la variation devant les différentes manières de poser la question en français, sera à l'écrit conscient de l'effet que produira son choix. Ainsi, la réflexivité accrue de Huston sur ses processus d'écriture d'une langue à l'autre permetelle au scripteur plurilingue de réfléchir à son propre maniement de plusieurs langues dans l'écriture ainsi qu'aux choix et non-choix stylistiques en lien avec «l'autorité » de la langue et/ou la norme linguistique imposée.

\section{Les textes écrits par les apprenantes}

Les participantes à l'étude sont des adultes plurilingues qui, pour la plupart, ont une connaissance à des degrés variés de quatre langues qu'elles utilisent avec des compétences diverses. Elles apprennent ou perfectionnent le français, dans ma classe de FLE niveau B2 selon le CECRL, pour des raisons diverses tels qu'augmenter leur chance de trouver un emploi en France ou à l'étranger, intégrer un programme de formation universitaire française, enseigner la langue dans leur pays d'origine. Très peu ont suivi un atelier d'écriture avant celui que je décris et qui s'est déroulé en 2009 et 2010. Dans ce qui suit, sont précisées entre parenthèses les langues comprises dans le répertoire plurilingue de chacune. 


\section{Vera (anglais, français, inuktitut, chinois)}

Vera, Canadienne, a vécu pendant deux ans dans le Grand Nord lors de ses premières années en tant qu'enseignante :

Curieusement, j'utilise quelques mots inuktitut, même 17 ans plus tard; les mots que mes élèves inuit utilisaient tout le temps. Par exemple, le mot "amaanatuq" tombé de mes lèvres de temps en temps quand je ne peux me rappeler le nom d'une chose. Parfois, au lieu de dire à mes enfants "venez-ici", je leur dis l'équivalent en inuktitut "kaigi". Et, elles le comprennent bien! (Vera, 2010, Pond Inlet)

Dans cet extrait, à la manière réflexive de Huston, Vera révèle son usage courant de mots inuktitut tel que amaanatuq et kaigi écrits entre guillemets. Les identités linguistiques de Vera se manifestent par sa compétence à apprendre et à transmettre les langues (à ses enfants, à ses élèves et à ses lecteurs). En effet, non seulement, elle utilise l'alternance codique en inuktitut dans son texte de manière tout à fait fluide, mais elle en donne une explication à son lecteur francophone afin qu'il puisse en comprendre la signification.

\section{Thich (vietnamien, anglais, chinois, français)}

Thich, Vietnamienne, a rejoint son mari en France avec l'intention de continuer des études supérieures :

Notre phrase est construite de façon identique : le sujet + le verbe + le mot qui indique le temps + l'objet. Il n'y a pas de conjugaison du futur ou du passé dans notre langue. Par exemple, le vietnamien dit “Tôi đã ăn cơm ", le chinois dit “我吃 饭了”, c'est-à-dire “j'ai déjà mangé ". Les mots “ đã " et “了” indique le temps du passé (Thich, 2010, Mes langues).

Thich explique que le chinois et le vietnamien se construisent de la même manière en ce qui concerne les temps : une particule temporelle est utilisée pour signifier le temps du passé. En tant que locutrice-apprenante de plusieurs langues, elle prend un rôle d'expert de ses langues par le biais de réflexions métalinguistiques. Elle utilise les caractères respectifs de chaque langue tout en conservant le français comme structure de base. Son identité en tant que locutrice plurilingue se manifeste par sa compétence à transmettre un savoir sur différents systèmes langagiers que le lecteur français ne connaîtra peut-être pas. L'alternance codique en vietnamien et en chinois écrite entre guillemets a une fonction métalinguistique à caractère didactique, soit, à la manière de Chraïbi, une volonté de faire comprendre en expliquant le fonctionnement de deux langues asiatiques, leurs similarités et leurs différences avec le français.

\section{Nati (géorgien, russe, anglais, français)}

Nati, Géorgienne, a passé plusieurs années en Russie avant de s'installer en France où elle a entrepris un Master en relations internationales :

Qu'est-ce qui pourrait être plus facile à dire que quelques mots sur soi... ? C'est évident! Mais, quand je commence à écrire: «je suis...", mon Babylon me fait perdre tous les mots. Les quatre personnes à l'intérieur de moi commencent à parler toutes ensemble dans une langue différente. Elles se bousculent en prétendant atteindre la page la première. So, finalement je dirais : $\mathrm{M} \mathrm{e} \mathrm{j}$ a $\mathrm{z}$ o v u t Nati. I love ma famille, $\mathrm{t} \mathrm{s} \mathrm{h} \mathrm{o} v \mathrm{r} \mathrm{e} \mathrm{b} \mathrm{a}$, et j'espère que c'est mutuel (Nati, 2009, Biographie).

Lorsque Nati écrit à propos de ses langues, elle transforme le nom d'une ville en un phénomène personnel qui fait référence à la tour de Babel, sa tour de Babel, c'est-à-dire, pour elle, son chaos linguistique. Son Babylon est un lieu dans lequel les langues sont personnifiées, à la manière de Huston. Parler de soi, c'est parler de ses langues et de l'équilibre et du déséquilibre qu'elles convoquent, de l'absence et de la présence des locuteurs porteurs de ces langues. Elle écrit en quatre langues : d'abord l'interjection so 
en anglais, puis le français, langue cible, ensuite elle se présente en russe $m$ e $n j$ a $z$ o $v u t$ (je m'appelle), parle de sa famille en anglais, fait référence à la Géorgie $T s h o v r$ e $b a$, et revient en français pour l'excipit. Elle enchâsse les quatre langues qui font partie de son répertoire langagier et font référence à la famille, l'histoire, les racines, mais aussi l'ouverture sur le monde. Ces langues entremêlées, des fils à tisser, nomment l'attachement aux valeurs ancestrales tout en croisant passés et futurs possibles.

\section{Discussion}

31 Il semblerait que les extraits de textes d'auteurs étudiés en classe ont permis aux apprenantes de mettre en lumière, dans des écrits courts, leur répertoire et leurs identités plurilingues. On observe, en effet, des textes d'apprenantes qui, bien que principalement rédigés en français - la langue d'apprentissage -, font appel à d'autres langues. Ces autres langues apparaissent dans leur graphie originale (comme le chinois, le vietnamien) et/ou dans des graphies autres que celles dans lesquelles elles seraient écrites dans leur contexte d'origine (le cyrillique et l'inuktitut écrits en alphabet latin). Les textes composés mettent ainsi en évidence la compétence plurilittératiée des apprenantes, l'utilisation de l'alternance codique et du tissage codique, mais aussi l'intertextualité existante entre les différents types de récits proposés en atelier. On pourrait donc dire que les auteurs plurilingues, tels Chraibi, Mouawad et Huston, en mettant en exergue leur répertoire plurilingue, offrent une forme de légitimation à l'utilisation de plusieurs langues et variétés de langues que les apprenantes de langues n'utiliseraient probablement pas si le contexte didactique ne le permettait pas explicitement.

D'un point de vue didactique, il s'agirait d'envisager les apprenants comme des individus plurilingues pour qui leurs identités et leur palette de langues sont propices à leur développement plurilittératié. Les apprenants utilisent, en effet, toutes les ressources à disposition, y compris la modalité graphique des langues, pour apprendre et/ou perfectionner une autre langue. Par ailleurs, il serait essentiel de considérer l'apport extrêmement riche de textes d'auteurs ayant navigué (entre) plusieurs langues, en mettant en lumière leurs stratégies textuelles et multimodales et en les reconnaissant, eux aussi, comme étant plurilingues. On ne peut que le constater : les apprenantes, en utilisant leurs langues dans des textes en français, bousculent la norme du texte monolingue essentiellement pratiqué dans les centres d'apprentissage de langue française.

\section{BIBLIOGRAPHIE}

CANAgARAJAH, A. Suresh. (2006). « The place of World Englishes in Composition: Pluralization Continued », College Composition and Communication, 57, nº4, pp. 586-619. 
CANAGARAJAH, A. Suresh (2011). « Codemeshing in academic writing: Identifying teachable strategies of translanguaging ", Modern Language Journal, 95, pp. 401-417.

CANDELIER, Michel (2008). « Approches plurielles, didactiques du plurilinguisme : le même et l'autre », Les cahiers de l'Acedle, 5, nำ1, pp. 65-90.

CHRAїBI, Driss (1998). Vu, lu, entendu. Paris : Denoël.

CICUREL, Francine (2000). « Avant-propos », ELA, 119, pp. 261-265.

COSTE, Daniel, MOORE, Danièle, Zarate, Geneviève (1997/2009). La compétence plurilingue et pluriculturelle. Strasbourg : Éditions du Conseil de l'Europe.

CUMMINS, Jim \& EARLY, Margaret (2011). Identity Texts - the Collaborative Creation of Power in Multilingual Schools. London : Institute of Education Press.

DAGENAIS, Diane \& MOORE, Danièle (2008). «Représentations des littératies plurilingues, de l'immersion en français et des dynamiques identitaires chez des parents chinois », Canadian Modern Language Review, 65, nº1, pp. 11-31.

DION, Robert, LÜSEBRINK, Hans-Jürgen, RIESZ, János (2002). Écrire en langue étrangère. Québec :

Éditions Nota Bene.

GAUVIN, Lise (2000). Langagement. Montréal : Les Éditions Boréal.

GROSJEAN, François (1995). « Le bilinguisme et le biculturalisme : essai de définition », TRANEL, 19, pp. 13-41.

HUSTON, Nancy (1999). Nord Perdu. Arles : Actes Sud/Leméac.

HUSTON, Nancy \& SEBBAR, Leïla (1986). Lettres parisiennes - Histoires d'exil. Paris : J’ai lu.

MATHIS, Noëlle (2013). Identités plurilingues et création textuelle en français langue étrangère : une approche sociolinguistique d'ateliers d'écriture plurielle. [on-line], Thèse de doctorat. Université d'Avignon \& Simon Fraser Université.

<URL : http://www.theses.fr/2013AVIG1121>.

MATHIS, Noëlle (2014). «Quand le multiple s'y mêle : récit et réflexivité d'une animatrice d'ateliers d'écriture entre plusieurs langues ». Actes du colloque Aix-Marseille Université » Former aux ateliers d'écriture à l'université, vingt ans après : métamorphoses, pratiques, perspectives ", [on-line], $<\mathrm{URL}:$ http://duecriture.canalblog.com>.

MOORE, Danièle \& BROHY, Claudine (2013). «Identités plurilingues et pluriculturelles » in Jacky Simonin \& Sylvie Wharton (éds.), Sociolinguistique du contact : Dictionnaire encyclopédique des termes et concepts. Lyon : ENS-Éditions, pp. 289-315.

MOORE, Danièle (2006). Plurilinguismes et école. Paris : Didier.

MOUAWAD, Wajdi (2008). Seuls. Chemin, texte et peintures. Arles : Actes Sud.

ROBIN, Régine (2003). Le deuil de l'origine. Une langue en trop la langue en moins. Paris : Éditions Kimé. VAN DEN AVENNE, Cécile (2013). « Écrits plurilingues » in Jacky Simonin \& Sylvie Wharton (éds.), Sociolinguistique du contact : Dictionnaire encyclopédique des termes et concepts. Lyon : ENS-Éditions, pp. 245-262. 


\section{NOTES}

1. Moore (2006: 118) propose le terme « pratiques plurilittératiées » pour définir « toute instance au cours de laquelle la communication se construit dans plus de deux langues dans et autour de l'écrit ». La notion de littératie se comprend comme désignant à la fois les pratiques sociales et les conceptions liées à la lecture et à l'écriture par les acteurs sociaux précisément qui les mettent en œuvre, et prend en compte les contextes de production / réception des pratiques langagières des individus plurilingues, considérés comme des acteurs sociaux, en rapport au pouvoir.

2. Les identités plurilingues sont une catégorie de l'identité (individuelle et/ou collective). Elles s'expriment au travers de l'usage que fait un locuteur de ses langues, et au travers de ses discours sur celles-ci. Elles sont marquées par l'instabilité et l'ambivalence. Elles se révèlent de manière différente selon les choix des locuteurs à l'intérieur de l'ensemble de possibles, choix qui dépendent, entre autres, des trajectoires de vie individuelle, des catégories sociales et linguistiques à disposition et des interprétations que donne l'individu aux circonstances locales dans lesquelles il est amené à négocier la différence et à signaler ses affiliations (Moore \& Brohy, 2013 : 297).

\section{RÉSUMÉS}

Lors d'ateliers d'écriture plurilingue à l'université avec des apprenants adultes de français langue étrangère, j'utilise des extraits de textes d'auteurs qui s'exercent à rendre transparent le maniement de leurs diverses langues. Dans cet article, je tente de montrer comment ces textes offrent une forme de légitimation aux apprenants afin que ces derniers puissent également utiliser leur répertoire composé de plusieurs langues et leurs identités plurilingues au profit de leur apprentissage langagier.

During creative writing workshops at university with French as a Second Language adult students, I use the works of published authors who navigate amongst several languages in their writing. In this article, I attempt to show how these texts offer a kind of legitimation to students so that they also use their plurilingual repertoire and identities in their literacy practices as a way to improve language competencies.

\section{INDEX}

Mots-clés : atelier d'écriture plurilingue, identités et répertoire plurilingue

Keywords : plurilingual writing workshops, plurilingual identity and repertoire 
AUTEUR

NOËLLE MATHIS

LIDILEM

mathisnoelle[at]yahoo.fr 